\title{
Population-based analysis of small cell carcinoma of the esophagus using the SEER database
}

\author{
Jiangyan $\mathrm{Li}^{1 \#}$, Jun $\mathrm{Ma}^{2 \#}$, Hao Wang ${ }^{1 \#}$, Jianyong Niu' ${ }^{2}$ Lin Zhou $^{1}$ \\ ${ }^{1}$ Department of Laboratory Medicine, Changzheng Hospital, Naval Medical University, Shanghai, China; ${ }^{2}$ Department of Cardiothoracic Surgery, \\ Heji Hospital, Changzhi Medical College, Changzhi, China \\ Contributions: (I) Conception and design: L Zhou, J Li; (II) Administrative support: J Niu, J Ma; (III) Provision of study materials or patients: J Li, \\ J Ma, H Wang; (IV) Collection and assembly of data: J Li, J Ma, H Wang; (V) Data analysis and interpretation: J Niu, L Zhou; (VI) Manuscript \\ writing: All authors; (VII) Final approval of manuscript: All authors. \\ \#These authors contributed equally to this work. \\ Correspondence to: Lin Zhou. Department of Laboratory Medicine, Changzheng Hospital, Naval Medical University, Shanghai, China. Email: \\ lynnzhou36@163.com; Jianyong Niu. Department of Cardiothoracic Surgery, Heji Hospital, Changzhi Medical College, Changzhi, China. Email: \\ $330591615 @ q q . c o m$.
}

\begin{abstract}
Background: Small cell cancer (SmCC) of the esophagus is a rare malignancy with an aggressive behavior associated with poor survival. The present study aims to determine the clinicopathological characteristics, therapeutic and prognosis.

Methods: Patients with SmCC of the esophagus, diagnosed from 1975 to 2016, were identified from the Surveillance, Epidemiology, and End Results (SEER) database. The clinicopathological characteristics were described and the prognostic factors were further determined using Cox regression analysis.

Results: The median overall survival (mOS) of all 515 patients with SmCC of the esophagus was 7.0 months, and the 1-, 2-, and 5-year survival rates were $31.5 \%, 14.7 \%, 6.00 \%$, respectively. Patients with chemoradiotherapy (mOS: 12.0 months) had better prognosis than those receiving surgery alone (mOS: 12.0 vs. 4.0 months). The patients receiving surgery combined with chemoradiotherapy had longest survival time (mOS: 19.0 months), followed by patients receiving surgery combined with chemotherapy (14.0 months). The multivariate Cox survival analysis demonstrated that older age, distant metastases were independent prognostic factors. The use of surgery, chemotherapy, radiotherapy were independent favorable prognostic factors $(\mathrm{P}<0.05$ for all).

Conclusions: SmCC of the esophagus is uncommon, older age and distant metastases were independently associated with poor survival. Chemotherapy could provide significant clinical benefit for those patients, especially chemoradiotherapy and surgery combined with chemotherapy.
\end{abstract}

Keywords: Small cell carcinoma of the esophagus; esophageal cancer; outcome; Surveillance, Epidemiology, and End Results database (SEER database)

Submitted Mar 21, 2020. Accepted for publication Jun 02, 2020.

doi: $10.21037 /$ jtd-20-1428

View this article at: http://dx.doi.org/10.21037/jtd-20-1428

\section{Introduction}

Primary extra-pulmonary small cell cancer (SmCC) is a rare pathologic entity, and the esophagus is the most frequently reported site in the digestive tract (1). Alike to its pulmonary counterpart, SmCC of the esophagus is notoriously aggressive and characterized by early systemic dissemination and poor prognosis, regardless of the methods of treatment $(2,3)$. Histologically, SmCC is characterized by a neuroendocrine-like architectural pattern, with peripheral palisading and rosette formation within the tumors. Similar to small cell lung cancer, SmCC of the esophagus is diagnosed based on the immunohistochemical 
staining for common neuroendocrine markers such as NSE, CD56, CgA, Syn (4). With the development of diagnostic capabilities such as immunohistochemical analysis, SmCC of esophagus could be readily diagnosed.

SmCC of the esophagus has been regarded as having an aggressive biological potential with poor prognosis. Due to its rarity, most previous studies have focused on case reports or series, the clinicopathological characteristics and the optimal clinical management has not yet been defined (5-8). Meanwhile, because the follow-up studies with long-term survival are limited, the factors affecting overall survival in those patients also remained unclear. Thus, the current retrospective study aimed to describe the clinicopathological characteristics and outcome of this rare disease base on the data of all patients with SmCC of the esophagus registered in the Surveillance, Epidemiology, and End Results (SEER) database from 1975 to 2016 . We present the following article in accordance with the STROBE reporting checklist (available at http://dx.doi.org/10.21037/jtd-20-1428).

\section{Methods}

\section{Data source and participants}

The SEER database is a population-based cancer registry that covers $28 \%$ of the population in the United States. The database 'SEER 18 Regs Custom Data with additional treatment fields, Nov 2018 Sub (1975-2016)' was searched for all patients diagnosed with SmCC of the esophagus using SEER*STAT 8.3.6 software. Patients with SmCC of esophagus were identified using "ICD-O 8041, 8041" between 1975 and 2016. Clinicopathological characteristics including age at diagnosis, race, sex, site of primary tumor, pathological grade, SEER historic stage classification, TNM stage, were extracted. Survival status as well as overall survival time were identified. Overall survival was defined as the interval from diagnosis to death or last follow-up. Treatment methods included surgery, chemotherapy, and radiation therapy, and different treatment strategy were combined to form seven treatment strategies, including: no treatment, surgery $(\mathrm{S})$, chemotherapy $(\mathrm{C})$, radiotherapy (R), surgery combined with chemotherapy (SC), surgery combined with radiotherapy (SR), chemoradiotherapy (CR), and surgery combined with chemoradiotherapy (SCR).

\section{Statistical analysis}

The clinicopathological characteristics were presented using counts and percentages. Kaplan-Meier method and log-rank tests were used to determine the influence of each variable on survival. Univariate and multivariate Cox regression survival analysis were also conducted to estimate the predictive factors and their weights, which was summarized using estimates and $95 \%$ confidence intervals for the hazard ratio. All statistical analysis was performed using SPSS software (version 23.0, IBM, USA).

\section{Results}

A total of 515 patients diagnosed with SmCC of the esophagus were identified from SEER database between 1975 and 2016. The demographics and clinicopathological characteristics of these patients are present in Table 1. Two hundred and two patients were female and 313 were male. The average age at diagnosis was 68.6 years. The lesions of primary tumor in the most patients $(76.0 \%)$ were located in middle/lower third of esophagus. A majority $(97.5 \%)$ of those patients had poorly or undifferentiated pathological grade. Among those patients with TNM stage information, more than half of patients had lymph node metastases (112/197) or distant metastases (128/227) and $128 / 207$ patients were diagnosed with TNM-IV stage. As for treatment regimen, most patients $(462 / 515)$ did not undergo surgery, and $45.4 \%$ patients received radiotherapy, $62.9 \%$ received chemotherapy. Most patients received chemoradiotherapy (155/515) and chemotherapy alone (139/515).

The median overall survival (mOS) of all 515 patients SmCC of the esophagus was 7.0 months (95\% CI, 6.0-9.0, Figure $1 A$ ). The OS rate by 6, 12, 24 months were $53.6 \%$, $31.5 \%, 14.7 \%$, respectively. Only $6.00 \%$ patients could survive exceed 5 years. Kaplan-Meier survival analysis revealed patients with distant stage had poorest prognosis, but no significant difference could be observed between patients with regional stage and localized stage (Figure $1 B$ ). Similarly, patients with advanced stage (IV) had much worse prognoses than those with early stage (I/II/III), but no difference among those patients without distant metastases (Figure 1C). The stratification analysis showed that older patients ( $>68$ years) had worse prognosis than ( $\leq 68$ years) $(\mathrm{P}<0.01)$. According AJCC-TNM stage classification, the prognoses did not become much worse with increasing tumor stage and lymph node invasion ( $\mathrm{P}>0.01$ for all, Figure $2 A, B, C$ ), but $M$ category is associated with the overall survival in patients with SmCC of the esophagus (Figure 2D). Besides, no significant association of other variables and survival 
Table 1 Clinicopathological characteristics of 515 patients with small cell carcinoma of esophagus

\begin{tabular}{|c|c|}
\hline Characteristics & Number \\
\hline Age (year) & $68.6 \pm 11.8$ \\
\hline \multicolumn{2}{|l|}{ Gender } \\
\hline Female & 202 \\
\hline Male & 313 \\
\hline \multicolumn{2}{|l|}{ Ethnicity } \\
\hline White & 404 \\
\hline Black & 81 \\
\hline $\begin{array}{l}\text { Other (American Indian/AK Native, Asian/ } \\
\text { Pacific Islander) }\end{array}$ & 30 \\
\hline \multicolumn{2}{|l|}{ Pathological differentiation } \\
\hline Well & 3 \\
\hline Moderately & 5 \\
\hline Poorly & 127 \\
\hline Undifferentiated & 189 \\
\hline Unknown & 191 \\
\hline \multicolumn{2}{|l|}{ Summary stage } \\
\hline Distant & 259 \\
\hline Regional & 80 \\
\hline Localized & 81 \\
\hline Unstaged & 95 \\
\hline \multicolumn{2}{|l|}{ Primary site } \\
\hline Cervical esophagus & 10 \\
\hline Upper third of esophagus & 36 \\
\hline Middle third of esophagus & 130 \\
\hline Lower third of esophagus & 218 \\
\hline Overlapping lesion of esophagus & 27 \\
\hline Unknown & 94 \\
\hline \multicolumn{2}{|l|}{ Marital status } \\
\hline Married & 279 \\
\hline Single & 61 \\
\hline Divorced/widowed/separated & 149 \\
\hline Unknown & 26 \\
\hline \multicolumn{2}{|l|}{ Tumor size } \\
\hline $\mathrm{T} 1$ & 61 \\
\hline $\mathrm{T} 2$ & 5 \\
\hline Т3 & 42 \\
\hline
\end{tabular}

Table 1 (continued)
Table 1 (continued)

\begin{tabular}{lc}
\hline Characteristics & Number \\
\hline T4 & 39 \\
Unknown & 368
\end{tabular}

Lymph node metastases

NO

N1

112

Unknown

318

Distant metastases

MO

99

M1

128

Unknown

288

TNM stage

I

27

II

20

III

32

IV

128

Unknown

308

Surgery

Yes

41

No

462

Unknown

12

Chemotherapy

Yes

324

No/unknown

191

Radiotherapy

Yes

No

Treatment strategy

Non-treatment

Chemotherapy alone

139

Radiotherapy alone

50

CR

155

Surgery alone $\quad 12$

SC

8

SR

4

SCR

17

16 patients have no information on treatment strategy. SC, surgery combined with chemotherapy; SR, surgery combined with radiotherapy; $\mathrm{CR}$, chemoradiotherapy; SCR, surgery combined with chemoradiotherapy. 

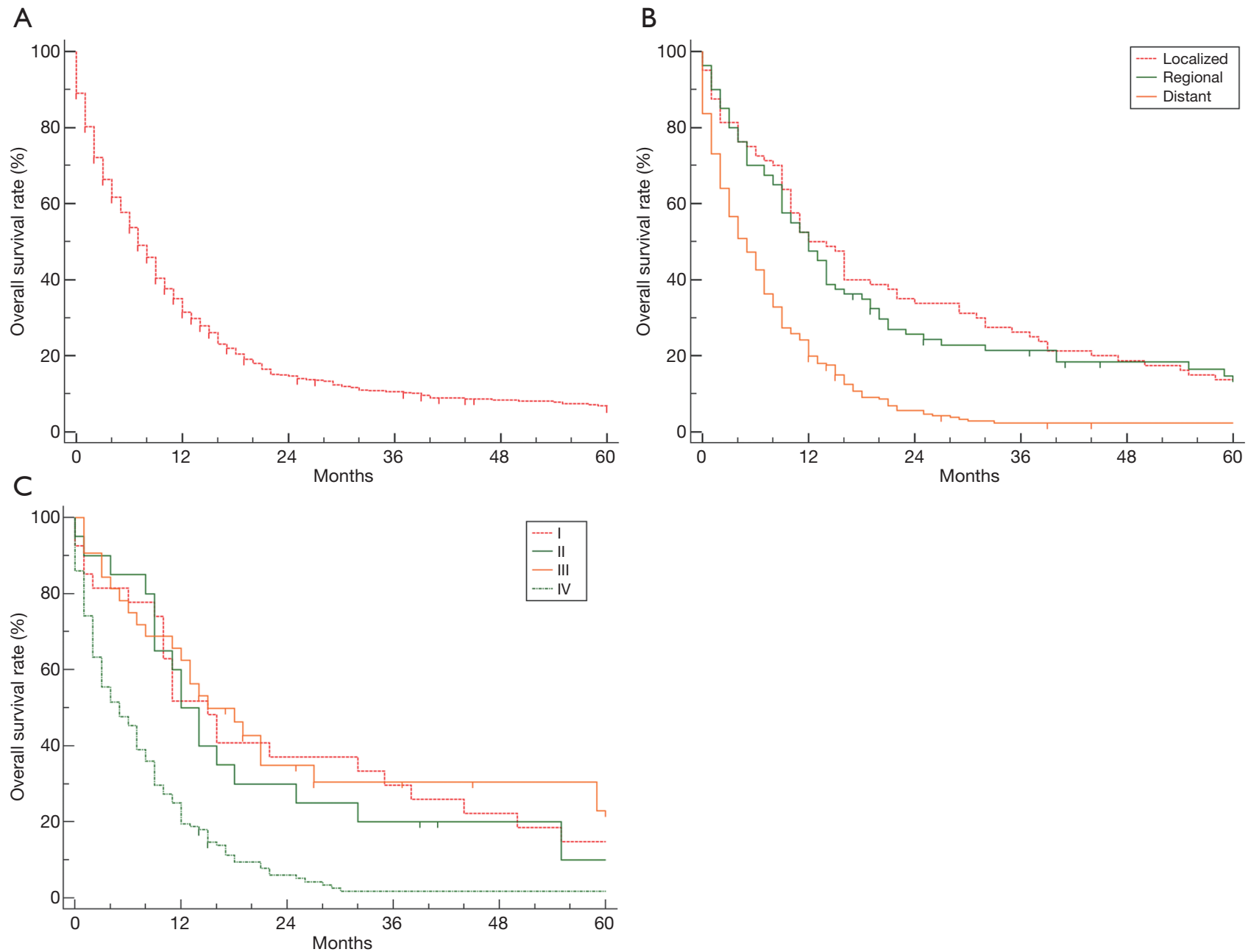

Figure 1 Overall survival of patients with SmCC of the esophagus. (A) Overall survival of all 515 patients; (B) overall survival stratified by SEER historic stage; (C) overall survival stratified by TNM stage. SmCC, small cell cancer; SEER, Surveillance, Epidemiology, and End Results.

could be observed including gender $(\mathrm{P}=0.53)$, race $(\mathrm{P}=0.40)$, site of primary tumor $(\mathrm{P}=0.26)$, etc.

One hundred and fourteen patients with SmCC of the esophagus did not receive any treatments, who also had shortest overall survival (mOS: 1.0 months). Patients with chemoradiotherapy (mOS: 12.0 months) had better prognosis than those receiving surgery alone (mOS: 4.0 months), only chemotherapy alone (mOS: 9.0 months) or radiotherapy alone (mOS: 4.0 months) (Figure $3 A$ ). Among 41 patients who received surgery, the patients receiving surgery combined with chemoradiotherapy had longest overall survival of patients with SmCC of the esophagus (mOS: 19.0 months), followed by patients receiving surgery combined with chemotherapy (mOS:
14.0 months), but no significantly statistical difference due to the small sample size (Figure 3B).

Table 2 summarizes the univariate and multivariate survival analysis for SmCC of the esophagus patients with respect to age at diagnosis, sex, race, pathological grade, stage of disease, treatment strategy. Univariate Cox survival analysis showed that age, distant metastases as well as treatment strategy were significantly associated with prognosis $(\mathrm{P}<0.05$ for all, Table 2). Subsequently, the multivariate Cox survival analysis demonstrated that older age ( $>68$ years) and distant metastases were independent factors for poor prognosis (HR $=1.33,95 \%$ CI, 1.10-1.61, HR $=2.28,95 \%$ CI, 1.68-3.08; Table 2). The use of surgery, chemotherapy, radiotherapy were independent 

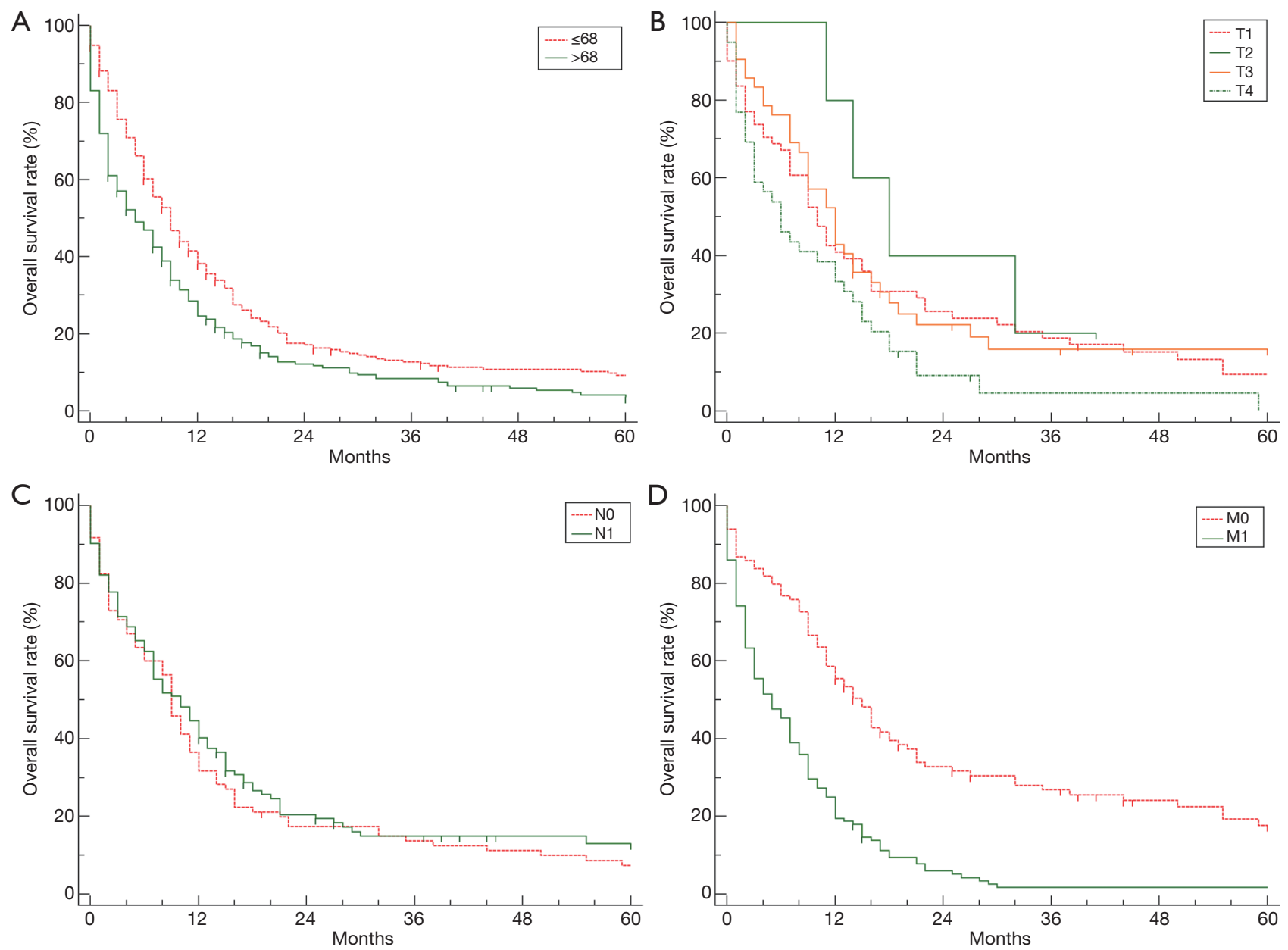

Figure 2 Overall survival for SmCC of the esophagus patients stratified by clinicopathological characteristics. (A) Age; (B) T category; (C) $\mathrm{N}$ category; (D) M category. SmCC, small cell cancer.

favorable prognostic factor for good prognosis. Surgery combined with chemoradiotherapy, surgery combined with chemotherapy as well as chemoradiotherapy could decrease risk of death for patients with SmCC of the esophagus by $77 \%, 81 \%, 77 \%$, respectively.

\section{Discussion}

Primary small cell carcinoma (SmCC) of the esophagus is a rare malignancy, accounting for $0.05-4 \%$ of all esophageal cancers (9-11). Although no predisposing risk factor has been identified with SmCC of the esophagus, some association with smoking, alcohol consumption and Barrett's disease have been reported previously. However, the clinicopathological characteristics and survival of this disease are not fully defined due to its rarity. In the present study, we conducted a retrospective study based on the data of 515 patients diagnosed with SmCC of the esophagus from SEER database to describe the clinicopathological characteristics and prognosis of this rare disease.

Previous studies reported that the lesion of patients with SmCC of the esophagus is more frequently located in the mid-lower esophagus and upper esophageal localization has been seen in approximately $5 \%$ of cases $(8,12,13)$. In consistent with this data, our study showed that the lesions of 348/421 cases were located in mid-lower esophagus. The affected patients were usually in the sixth decade of life, with an age range of 40-70 years, with male predominance (5). Similar to those reported data, out study also found a predominance of males $(1.55: 1)$ with an average age of 68.6 years, which is also used as the cut-off value in terms of age for older patients and younger patients. The most 

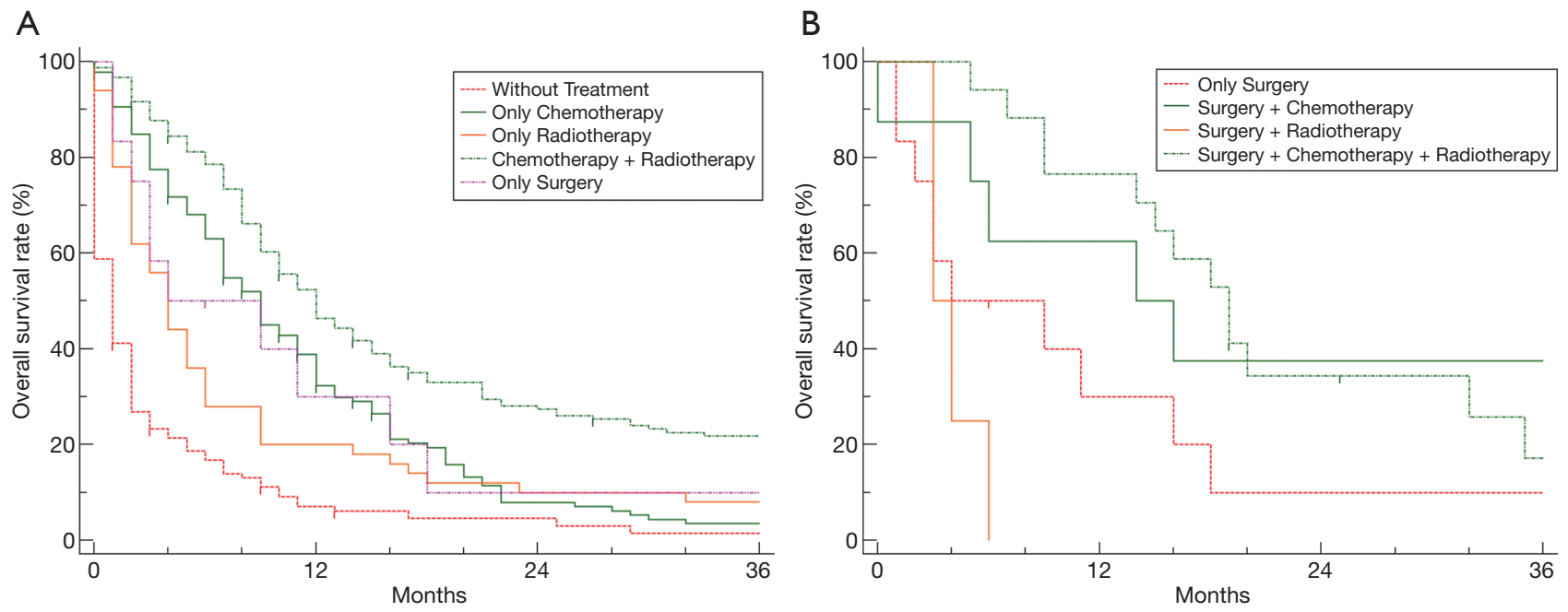

Figure 3 The effect of different treatment regimens on overall survival for patients with SmCC of the esophagus. SmCC, small cell cancer

common symptoms are dysphagia, anorexia, weight loss, reflux and chest pain $(14,15)$. Besides, paraneoplastic syndrome with ectopic secretion of hormones in those patients have been reported, although this is a rare finding (1). In 2013, Kukar conducted a retrospective study with 64,799 esophageal cancer patients (387 patients with SmCC of the esophagus) from SEER database between 1973 and 2009 to compare the clinical characteristic between SmCC and other esophageal cancer (16). Compared with non-SmCC esophageal cancer, patients with SmCC of the esophagus were similar in age and race but had a higher proportion of women, higher stage and less likely to undergo surgical resection (16).

SmCC often have an aggressive behavior associated with a higher metastatic potential whatever primary site $(2,3)$. For example, almost $56.6 \%$ of patients diagnosed with small cell lung cancer were staged with extensive-stage disease at initial diagnosis (2). Similarly, SmCC of the esophagus also had high rate of lymph node involvement and a high percentage of cases are diagnosed with distant metastasis (31-90\%) (1). In the present cohort, approximately $97.5 \%$ of cases had poor differentiation or undifferentiation. More than half of cases accompanied with lymph node (112/197) or distant metastases $(128 / 227)$ at diagnosis. Therefore, most patients present locally or regionally advanced disease at diagnosis, whatever SEER historic stage (distant stage: 259/420) or AJCC-TNM stage (IV stage: 128/207). The prognosis of this rare malignancy is poor, with reported median survival ranging from 7 to 19 months $(14,17)$. The mOS of patients in the present cohort is only 7.0 months, only $31.5 \%$ patients could survive exceed 2 years. In Kukar's study, the data showed that patients with SmCC of the esophagus had a worse survival than other esophageal cancer (16). In consistent with Kukar's study, our data also revealed that older age, distant metastases were independent prognostic factors.

Treatment regimens have not been established nor have been studies in the prospective randomized trials due to the low incidence rate of this rare disease. The reported cases of SmCC of the esophagus have been managed with various treatment methods including resection, radiotherapy, chemotherapy as well as combinations of them. Chemoradiotherapy is the prevailing management for bronchogenic SmCC, but its role in the treatment of patients with SmCC of the esophagus remains unclear. In Yau et al.'s study, the mOS was 8 months for those patients who were managed non-operatively by either chemotherapy and/or radiotherapy (7). Yau's conclusion suggested that satisfactory palliation could be achieved with chemoradiotherapy for patients with SmCC of the esophagus (7). In the present study, both radiotherapy and chemotherapy could improve the patients' prognoses. Chemotherapy had the biggest influence on prognoses for those patients that patients receiving chemotherapy had mOS of 11 months, compared with 2 months in individuals without chemotherapy. Surgical resection is a curative monotherapy for early squamous and adenocarcinoma of the esophagus, the role of esophagectomy has been controversial in the management of SmCC of the esophagus. Tanaka et al.'s study found the mOS was 
Table 2 Univariate and multivariate Cox proportional hazard analyses of the characteristics for overall survival in patients with small cell carcinoma of esophagus

\begin{tabular}{|c|c|c|c|c|c|}
\hline Factor & Category & \multicolumn{2}{|c|}{ Univariate } & \multicolumn{2}{|c|}{ Multivariate } \\
\hline \multirow[t]{2}{*}{ Age } & $\leq 68$ & Reference & & Reference & \\
\hline & $>68$ & $1.39(1.16-1.67)$ & $<0.01$ & $1.33(1.10-1.61)$ & $<0.01$ \\
\hline Gender & Female & Reference & & & \\
\hline \multirow[t]{3}{*}{ Race } & White & Reference & & & \\
\hline & Black & $1.11(0.87-1.42)$ & 0.40 & & \\
\hline & Other & $0.75(0.50-1.11)$ & 0.14 & & \\
\hline Pathological differentiation & Well/moderately & Reference & & & \\
\hline \multirow{4}{*}{ Primary site } & Upper third of esophagus & $1.08(0.53-2.02)$ & 0.82 & & \\
\hline & Middle third of esophagus & $1.25(0.66-2.38)$ & 0.50 & & \\
\hline & Lower third of esophagus & $1.17(0.62-2.21)$ & 0.62 & & \\
\hline & Overlapping lesion of esophagus & $1.97(0.95-4.08)$ & 0.06 & & \\
\hline \multirow[t]{3}{*}{ Marital } & Married & Reference & & & \\
\hline & Single & $1.18(0.89-1.89)$ & 0.24 & & \\
\hline & Divorced/widowed/separated & $1.24(1.02-1.52)$ & 0.04 & & \\
\hline Summary stage & Localized & Reference & & & \\
\hline TNM & IV & $2.14(1.40-3.29)$ & $<0.01$ & & \\
\hline \multirow[t]{4}{*}{ Tumor size } & T1 & Reference & & & \\
\hline & $\mathrm{T} 2$ & $0.61(0.22-1.67)$ & 0.33 & & \\
\hline & T3 & $0.87(0.57-1.33)$ & 0.53 & & \\
\hline & T4 & $1.43(0.95-2.16)$ & 0.09 & & \\
\hline \multirow[t]{2}{*}{ Lymph node metastases } & No & Reference & & & \\
\hline & N1 & $0.86(0.64-1.15)$ & 0.31 & & \\
\hline \multirow[t]{2}{*}{ Distant metastases } & MO & Reference & & & \\
\hline & M1 & 2.42 (1.82-3.22) & $<0.01$ & $2.28(1.68-3.08)$ & 0.01 \\
\hline Surgery & Yes/no & $0.77(0.51-0.88)$ & 0.04 & $0.77(0.55-1.09)$ & 0.14 \\
\hline
\end{tabular}

Table 2 (continued) 
Table 2 (continued)

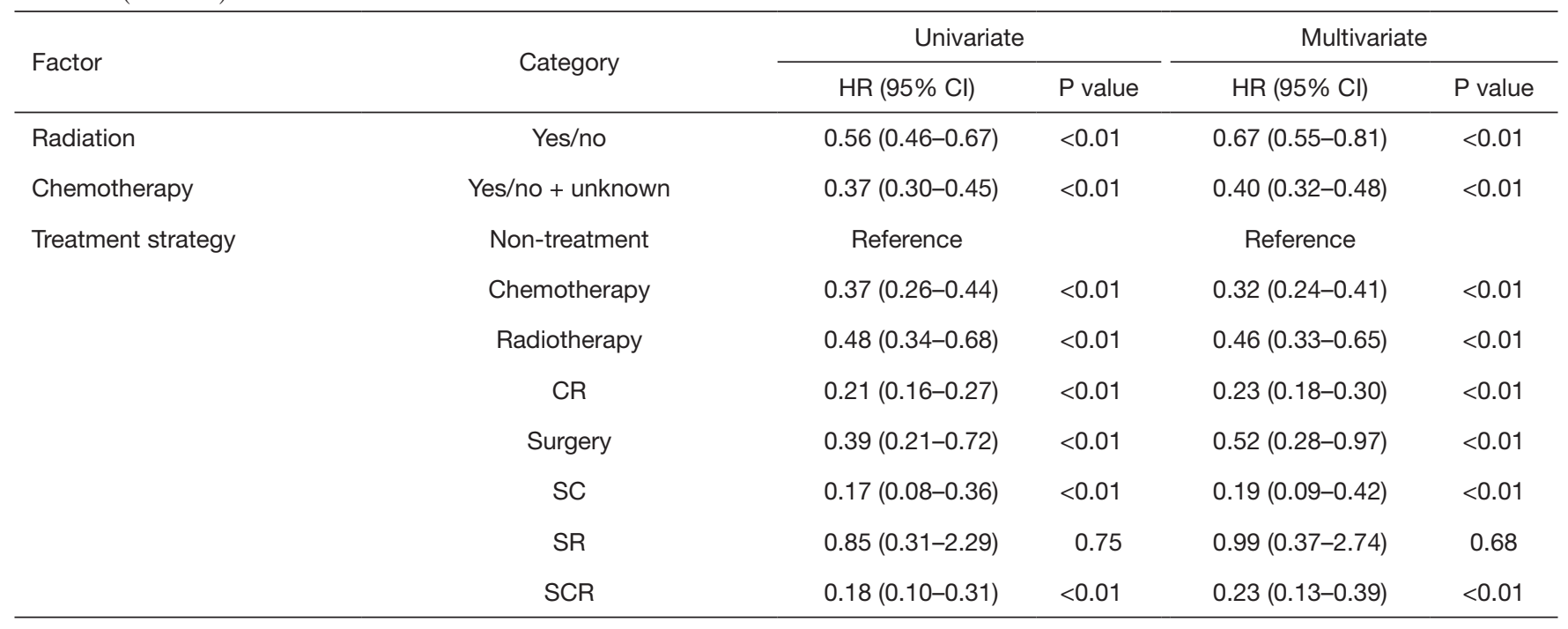

SC, surgery combined with chemotherapy; SR, surgery combined with radiotherapy; CR, chemoradiotherapy; SCR, surgery combined with chemoradiotherapy.

16 months after surgical management, esophagectomy with lymphadenectomy could result in a relatively better survival in some patients (6). Lieberman et al. found that the longest median survival time (28 months) was obtained in patients treated by esophagectomy in combination with chemotherapy in their review of 107 previously reported patients with SmCC of the esophagus (18). In another series reported by Law et al., one patient survived 5 years but only after curative-intent esophagectomy (19). In our study, the data showed the patients with chemoradiotherapy had better prognosis than those receiving surgery alone, chemotherapy alone or radiotherapy alone. In multivariate Cox survival analysis, chemotherapy could provide more clinical benefit for those patients, especially chemoradiotherapy and surgery combined with chemotherapy. For example, among 26 patients who survived exceed 5 years, 22 patients received chemoradiotherapy and only 4 patients underwent surgical management alone. Among 41 patients with surgery, 10 patients received radiotherapy after surgery, 11 patients received radiotherapy before surgery, and 20 patients received surgery alone. Survival analysis revealed no significant difference in overall survival among those three cohorts of patients $(\mathrm{P}=0.33)$, suggesting radiotherapy has no effect on prognosis for patients who received surgery. Meanwhile, 25 out of 41 patients had received chemotherapy after surgery. The survival analysis showed that patients receiving chemotherapy after surgery had better prognosis than those with surgery alone (mOS: 18.0 vs. $4.0 \mathrm{~m}, \mathrm{P}<0.01)$. In addition, only 41 patients underwent surgical treatment in the present cohort. We speculated that two reasons may contribute to this low surgery rate. First, the low surgery rate may be related with the tumor stage. For example, only 57 out of 515 patients were diagnosed with TNM-I stage or II stage, and most patients had lymph node or distant metastases at diagnosis. Second, chemoradiotherapy could yield much more clinical benefit than surgery, so physician would prefer chemoradiotherapy than surgery when they made clinical decision. However, the optimal treatment protocol needs to be explored in the future prospective randomized trial.

Obviously, there are several limitations that required clarification like other studies based on SEER database. First, several variable information was lacking, such as pathological differentiation, TNM stage, chemotherapy, etc. For example, there were 308 patients without TNM stage information, 318 patients had no recorded lymph node metastases, and 288 patients had no information on distant metastases. Although SEER summary stage could be an alternative classification for AJCC-TNM stage, there are still 95 patients with unknown SEER summary stage. Inadequate data of those variable limited the reliability and accuracy of characteristics description and prognostic analysis. Second, the lack of other variables such as performance status, gene alterations, type and duration of chemotherapy, chemotherapy sequence surgery, also limited the accuracy of prognosis analysis. Lastly, responses 
to treatment and recurrence rates could not be determined using these data from the SEER database.

In conclusion, we present the largest case series on SmCC of the esophagus based on the data from SEER database. The results showed that older age, distant metastases were independent prognostic factors for poor survival. Chemotherapy could provide significant clinical benefit for those patients, especially chemoradiotherapy and surgery combined with chemotherapy. However, conclusion drawn from a study based on SEER database must be tempered by the fact that several variables was lack. Therefore, these conclusions required to be validated in the future prospective study.

\section{Acknowledgments}

Funding: None.

\section{Footnote}

Reporting Checklist: The authors have completed the STROBE reporting checklist. Available at http://dx.doi. org/10.21037/jtd-20-1428

Conflicts of Interest: All authors have completed the ICMJE uniform disclosure form (available at http://dx.doi. org/10.21037/jtd-20-1428). The authors have no conflicts of interest to declare.

Ethical Statement: The authors are accountable for all aspects of the work in ensuring that questions related to the accuracy or integrity of any part of the work are appropriately investigated and resolved.

Open Access Statement: This is an Open Access article distributed in accordance with the Creative Commons Attribution-NonCommercial-NoDerivs 4.0 International License (CC BY-NC-ND 4.0), which permits the noncommercial replication and distribution of the article with the strict proviso that no changes or edits are made and the original work is properly cited (including links to both the formal publication through the relevant DOI and the license). See: https://creativecommons.org/licenses/by-nc-nd/4.0/.

\section{References}

1. Brenner B, Tang LH, Shia J, et al. Small cell carcinomas of the gastrointestinal tract: clinicopathological features and treatment approach. Semin Oncol 2007;34:43-50.

2. Govindan R, Page N, Morgensztern D, et al. Changing epidemiology of small-cell lung cancer in the United States over the last 30 years: analysis of the surveillance, epidemiologic, and end results database. J Clin Oncol 2006;24:4539-44.

3. Bai J, Zhao F, Pan S. Clinicopathological Characteristics and Survival of Small Cell Carcinoma of the Salivary Gland: a Population-Based Study. Cancer Manag Res 2019;11:10749-57.

4. Giannetta E, Guarnotta V, Rota F, et al. A rare rarity: Neuroendocrine tumor of the esophagus. Crit Rev Oncol Hematol 2019;137:92-107.

5. Nevárez A, Saftoiu A, Bhutani MS. Primary Small Cell Carcinoma of the Esophagus: Clinico- pathological Features and Therapeutic Options. Curr Health Sci J 2011;37:31-4.

6. Tanaka T, Matono S, Nagano T, et al. Surgical management for small cell carcinoma of the esophagus. Dis Esophagus 2010;23:502-5.

7. Yau KK, Siu WT, Wong DC, et al. Non-operative management of small cell carcinoma of esophagus. Dis Esophagus 2007;20:487-90.

8. Casas F, Ferrer F, Farrus B, et al. Primary small cell carcinoma of the esophagus: a review of the literature with emphasis on therapy and prognosis. Cancer 1997;80:1366-72.

9. Nicholson SA, Beasley MB, Brambilla E, et al. Small cell lung carcinoma (SCLC): a clinicopathologic study of 100 cases with surgical specimens. Am J Surg Pathol 2002;26:1184-97.

10. Shin SJ, DeLellis RA, Ying L, et al. Small cell carcinoma of the breast: a clinicopathologic and immunohistochemical study of nine patients. Am J Surg Pathol 2000;24:1231-8.

11. Sasajima K, Watanabe M, Ando T, et al. Serum neuronspecific enolase as a marker of small-cell carcinoma of the esophagus. J Clin Gastroenterol 1990;12:384-8.

12. Ku GY, Minsky BD, Rusch VW, et al. Small-cell carcinoma of the esophagus and gastroesophageal junction: review of the Memorial Sloan-Kettering experience. Ann Oncol 2008;19:533-7.

13. Pantvaidya GH, Pramesh CS, Deshpande MS, et al. Small cell carcinoma of the esophagus: the Tata Memorial Hospital experience. Ann Thorac Surg 2002;74:1924-7.

14. Hudson E, Powell J, Mukherjee S, et al. Small cell oesophageal carcinoma: an institutional experience and review of the literature. Br J Cancer 2007;96:708-11.

15. Sun KL, He J, Cheng GY, et al. Management of primary 
small cell carcinoma of the esophagus. Chin Med J (Engl) 2007;120:355-8.

16. Kukar M, Groman A, Malhotra U, et al. Small cell carcinoma of the esophagus: a SEER database analysis. Ann Surg Oncol 2013;20:4239-44.

17. Yun JP, Zhang MF, Hou JH, et al. Primary small cell carcinoma of the esophagus: clinicopathological and

Cite this article as: Li J, Ma J, Wang H, Niu J, Zhou L. Population-based analysis of small cell carcinoma of the esophagus using the SEER database. J Thorac Dis 2020;12(7):35293538. doi: $10.21037 /$ jtd-20-1428 immunohistochemical features of 21 cases. BMC Cancer 2007;7:38.

18. Lieberman MD, Franceschi D, Marsan B, et al. Esophageal carcinoma. The unusual variants. J Thorac Cardiovasc Surg 1994;108:1138-46.

19. Law SY, Fok M, Lam KY, et al. Small cell carcinoma of the esophagus. Cancer 1994;73:2894-9. 